\title{
Greedy Online Frequency Allocation in Cellular Networks
}

\author{
Wun-Tat Chan* Francis Y. L. Chin ${ }^{\dagger} \quad$ Deshi Ye ${ }^{\ddagger} \quad$ Yong Zhang ${ }^{\S}$ Hong Zhu ${ }^{\Uparrow}$
}

\begin{abstract}
The online frequency allocation problem for cellular networks has been well studied in these years. Given a mobile telephone network, whose geographical coverage area is divided into cells, phone calls are served by assigning frequencies to them, and no two calls emanating from the same or neighboring cells are assigned the same frequency. Assuming an online setting that the calls arrive one by one, the problem is to minimize the span of the frequencies used.

In this paper, we study the greedy approach for the online frequency allocation problem, which assigns the minimal available frequency to a new call so that the call does not interfere with calls of the same cell or neighboring cells. If the calls have infinite duration, the competitive ratio of greedy algorithm has a tight upper bound of $17 / 7$, which closes the gap of $[17 / 7,2.5)$ in $[3]$. If the calls have finite duration, i.e., each call may be terminated at some time, the competitive ratio of the greedy algorithm has a tight upper bound of 3 .
\end{abstract}

Keywords: Online Frequency Allocation, Competitive Analysis, Greedy Algorithm, Cellular Network

\section{Introduction}

Wireless Communication based on Frequency Division Multiplexing (FDM) technology is widely used in the area of mobile telephony. In the network of wireless communication, the geographic area is divided into small cellular regions [8] or cells as shown in Fig. 1, each containing one base station. Each base station serves the calls in its cell via radio frequencies, and base stations communicate with each other through a high-speed wired network. To avoid radio interference, the same frequency cannot be assigned to two different calls emanating from the same cell or neighboring cells. Since the frequency spectrum is a scarce resource, we should reuse the same frequency for different calls in the cells not close to each other. Efficient utilization of the available spectrum is very important to the frequency allocation problem $[1,3,4,6,7,9,10]$.

Frequency Allocation Problem. In this paper, we focus on the online version of the frequency allocation problem, in which a sequence $\sigma$ of calls arrive over time, where $\sigma=$ $\left(C_{1}, C_{2}, \ldots, C_{k}, \ldots,\right)$ and $C_{k}$ represents the cell from which the $k$-th call emanates. Each call $C_{k}$ must be assigned upon its arrival, without information about future calls $\left\{C_{i} \mid i>k\right\}$, a frequency $\mathcal{A}\left(C_{k}\right) \in Z^{+}$where $Z^{+}=\{1,2, \ldots\}$ of available frequencies, that is different from those of other

\footnotetext{
${ }^{*}$ Department of Computer Science, University of Hong Kong, Hong Kong, wtchan@cs.hku.hk

${ }^{\dagger}$ Department of Computer Science, University of Hong Kong, Hong Kong, chin@cs.hku.hk

${ }^{\ddagger}$ Department of Computer Science, University of Hong Kong, Hong Kong, yedeshi@cs.hku.hk

${ }^{\S}$ Department of Computer Science, University of Hong Kong, Hong Kong, yzhang@cs.hku.hk

`Department of Computer Science and Engineering, Fudan University, China, hzhu@fudan.edu.cn
} 


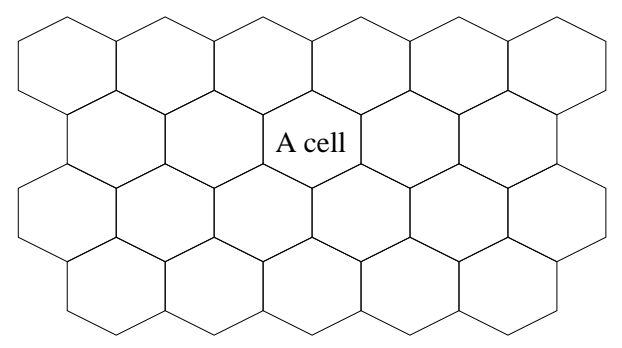

Figure 1: a description of cellular network

calls in the same cell or neighboring cells, i.e., $\mathcal{A}\left(C_{k}\right) \neq \mathcal{A}\left(C_{i}\right)$, where $i<k$ and $C_{i}$ is adjacent to $C_{k}$ or the same as $C_{k}$. The integer frequency once assigned to a call cannot be changed during the survival of this call. The online frequency allocation problem for cellular network (FAC for short) is to minimize the maximum assigned frequency, i.e. $\max \left\{\mathcal{A}\left(C_{k}\right) \mid k=1,2, \ldots, n\right\}$. If all the information of $C_{k}$ is known in advance, we call this problem off-line frequency allocation problem, which is NP-hard [9].

Two models of online frequency allocation problems will be investigated. The first model is that all the calls have infinite duration [3]. We call this model frequency allocation without deletion. The second model is that each call may be terminated at some time, i.e., each call is characterized by two parameters: arrival time and termination time. However, the termination time is not known when the call arrives online. We call this model frequency allocation with deletion.

Performance Measures. We use competitive analysis [2] to measure the performances of online algorithms. To serve all the calls in a given sequence $\sigma, \mathcal{A}(\sigma)$ denotes the highest frequency used by the online algorithm $\mathcal{A}$, and $\mathcal{O}(\sigma)$ denotes the highest frequency used by the optimal off-line algorithm.

The competitive ratio of algorithm $\mathcal{A}$ is defined as

$$
R_{\mathcal{A}}=\sup _{\sigma} \frac{\mathcal{A}(\sigma)}{\mathcal{O}(\sigma)}
$$

Known Results. Previous results have mainly focused on the without-deletion model. A simple strategy for frequency allocation problem is the fixed allocation assignment (FAA) [8], in which cells are partitioned into independent sets and independent sets are each assigned a separate set of frequencies. FAA gives an easy upper bound of 3 for FAC.

Another intuitive approach is the greedy algorithm (Greedy), which assigns the minimal available frequency to a new call so that the call does not interfere with calls of the same cell or neighboring cells. Caragiannis et al [3] proved that the competitive ratio of Greedy for FAC is at least $17 / 7$ and at most 2.5 .

In the with-deletion model, the general lower bound of competitive ratio is $2[5]$.

Our Contributions. In this paper, we analyze Greedy in both of the without-deletion and with-deletion models. In the without-deletion model, we tighten the upper bound of Greedy to $17 / 7$. In the with-deletion model, we prove that both the upper bound and the lower bound of Greedy are 3. Thus, 3 is the best possible competitive ratio for Greedy in with-deletion model. 


\section{Greedy in the Without-Deletion Model}

In this section we give a tighter analysis of Greedy and show that Greedy is 17/7-competitive for cellular networks, which matches the lower bound of Greedy as given in [3].

Theorem 1. Greedy for FAC has a competitive ratio of $17 / 7$ in the without-deletion model.

Proof. Suppose the highest frequency $h$ used by Greedy is assigned to a call from a cell $n_{0}$ after which no more calls are made. Let the six neighboring cells of $n_{0}$ be $n_{i}$ for $1 \leq i \leq 6$ in clockwise order as shown in Figure 2. Let $f^{*}$ be the maximum number of calls from any three adjacent cells. Let $C(N)$ denote the total number of calls from $N$ where $N$ can be an individual cell or a set of cells. Without loss of generality, we can assume that the three adjacent cells $n_{0}, n_{1}$, and $n_{2}$ together have $C\left(\left\{n_{0}, n_{1}, n_{2}\right\}\right)=f^{*}$ calls; otherwise, the adversary may initiate more calls from $n_{0}$ to get a larger $h$ and thereby increase the competitive ratio.

Let $C\left(n_{1}\right)=i, C\left(n_{2}\right)=i+j$, and $C\left(n_{0}\right)=i+k$, for some $i, j \geq 0$ and $k$ may be positive or negative. Hence, we have $f^{*}=3 i+j+k$. Consider another three adjacent cells, say $n_{0}, n_{2}$ and $n_{3}, C\left(\left\{n_{0}, n_{2}, n_{3}\right\}\right) \leq f^{*}$. Thus, we can assume $C\left(n_{3}\right)=i-c_{1}$ for some $c_{1} \geq 0$. Similarly, we can deduce the numbers of calls from other cells as follows.

$$
\begin{array}{cl}
C\left(n_{0}\right)=i+k, & C\left(n_{1}\right)=i, \quad C\left(n_{2}\right)=i+j, \quad C\left(n_{3}\right)=i-c_{1} \\
C\left(n_{4}\right)=i+j-c_{2}, & C\left(n_{5}\right)=i-c_{3}, \quad C\left(n_{6}\right)=i+j-c_{4}
\end{array}
$$

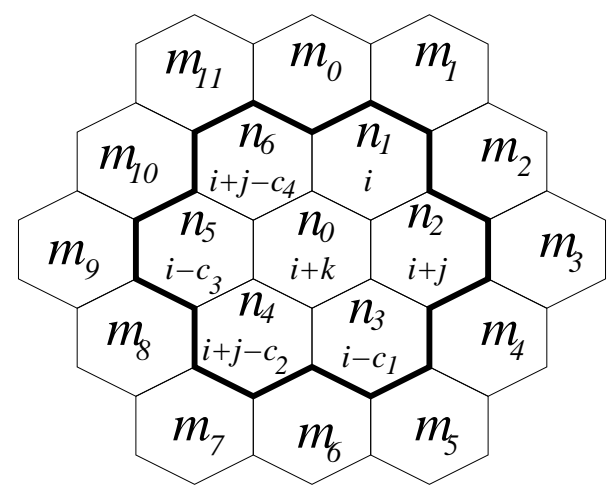

Figure 2: Cellular network for upper bound of Greedy

Let $d$ be the number of distinct frequencies among the frequencies assigned to the calls from $n_{0}$ and all $n_{0}$ 's neighbors. Let $r=\sum_{i=0}^{6} C\left(n_{i}\right)-d=7 i+3 j+k-\sum_{i=1}^{4} c_{i}-d$, i.e., $r$ is the total number of calls with "reused" frequencies in these seven cells. Recall that $h$ is the highest frequency used by Greedy and $f^{*}$ is the maximum number of calls from any three adjacent cells. The competitive ratio of Greedy is at most $h / f^{*}$. In the following we prove that $h / f^{*} \leq 17 / 7$. Since $h=d=\sum_{i=0}^{6} C\left(n_{i}\right)-r=7 i+3 j+k-\sum_{i=1}^{4} c_{i}-r$ and $f^{*}=3 i+j+k$, it suffices to prove that

$$
\frac{7 i+3 j+k-\sum_{i=1}^{4} c_{i}-r}{3 i+j+k} \leq \frac{17}{7}
$$


or after rewriting the inequality,

$$
2 i+10 k+7 \sum_{i=1}^{4} c_{i}+7 r \geq 4 j .
$$

This inequality is proved in the following lemma.

Lemma 2. $2 i+10 k+7 \sum_{i=1}^{4} c_{i}+7 r \geq 4 j$.

Proof. Let $M$ denote the set of cells, excluding $n_{0}$, that are neighbors of one of the cells $n_{i}$ for $1 \leq i \leq 6$ but not the cells $n_{i}$ themselves. Assume the cells in $M$ are labeled as $m_{i}$ for $0 \leq i \leq 11$ in clockwise order, starting with $m_{0}$ which is the neighbor of both $n_{1}$ and $n_{6}$ (Figure 2).

Let $s_{i}$ denote the total number of calls from cells in $M$ which are the neighbors of $n_{i}$. Precisely, $s_{i}=C\left(\left\{m_{2 i-2}, m_{2 i-1}, m_{2 i}\right\}\right)$. Note that the indices are in modulo 12 , e.g., $s_{6}=$ $C\left(\left\{m_{10}, m_{11}, m_{0}\right\}\right)$. We can deduce the value $s_{i}$ for $1 \leq i \leq 6$ as follows. For example, where $s_{4}$ is considered, we have $C\left(m_{6}\right) \leq f^{*}-C\left(\left\{n_{3}, n_{4}\right\}\right)=i+k+c_{1}+c_{2}$ because $m_{6}, n_{3}$ and $n_{4}$ are three adjacent cells and similarly $C\left(\left\{m_{7}, m_{8}\right\}\right) \leq f^{*}-C\left(n_{4}\right)=2 i+k+c_{2}$. Thus $s_{4}=$ $C\left(\left\{m_{6}, m_{7}, m_{8}\right\}\right) \leq 3 i+2 k+2 c_{2}+c_{1}$. On the other hand, we have $C\left(m_{8}\right) \leq f^{*}-C\left(\left\{n_{4}, n_{5}\right\}\right)=$ $i+k+c_{2}+c_{3}$ and $C\left(\left\{m_{6}, m_{7}\right\}\right) \leq f^{*}-C\left(n_{4}\right)=2 i+k+c_{2}$, and hence $s_{4} \leq 3 i+2 k+2 c_{2}+c_{3}$. Since $s_{4}$ has to satisfy both inequalities, we have $s_{4} \leq 3 i+2 k+2 c_{2}+\min \left(c_{1}, c_{3}\right)$. Similarly, we can deduce the inequalities for other $s_{i}$ as follows.

$$
\begin{array}{ll}
s_{1} \leq 3 i+j+2 k, & s_{2} \leq 3 i+2 k, \\
s_{3} \leq 3 i+j+2 k+2 c_{1}, & s_{4} \leq 3 i+2 k+2 c_{2}+\min \left(c_{1}, c_{3}\right), \\
s_{5} \leq 3 i+j+2 k+2 c_{3}+\min \left(c_{2}, c_{4}\right), & s_{6} \leq 3 i+2 k+2 c_{4}+\min \left(0, c_{3}\right) .
\end{array}
$$

Let $H(N)$ denote the highest frequency assigned to calls from $N$ where $N$ can be an individual cell or a set of cells. In the following, we study the six different cases where $H\left(\left\{n_{1}, n_{2}, \ldots, n_{6}\right\}\right)=H\left(n_{i}\right)$ for $1 \leq i \leq 6$. In each case, we show that $2 i+10 k+7 \sum_{i=1}^{4} c_{i}+7 r \geq$ $4 j$.

Case 1: $\boldsymbol{H}\left(\left\{n_{1}, n_{2}, \ldots, n_{6}\right\}\right)=\boldsymbol{H}\left(\boldsymbol{n}_{1}\right)$ : By the greedy algorithm, when frequency $H\left(n_{1}\right)$ is assigned to a call from $n_{1}$, all the frequencies less than $H\left(n_{1}\right)$ must have been assigned to calls from $n_{1}$ or neighbors of $n_{1}$. Those frequencies in $n_{3}, n_{4}$, and $n_{5}$ but not duplicated in $n_{1}, n_{2}$ and $n_{6}$ must be assigned to calls from $m_{0}, m_{1}$ or $m_{2}$, which are the other neighbors of $n_{1}$. Hence, we have (from Eq. (1) and (2))

$$
\begin{aligned}
& C\left(\left\{n_{3}, n_{4}, n_{5}\right\}\right)-r \leq s_{1}=3 i+j+2 k \\
\Rightarrow \quad & 3 i+j-c_{1}-c_{2}-c_{3}-r \leq 3 i+j+2 k \\
\Rightarrow \quad & 2 k+c_{1}+c_{2}+c_{3}+r \geq 0 .
\end{aligned}
$$

We further consider three sub-cases: $H\left(\left\{n_{2}, n_{4}, n_{6}\right\}\right)=H\left(n_{2}\right), H\left(\left\{n_{2}, n_{4}, n_{6}\right\}\right)=H\left(n_{4}\right)$, and $H\left(\left\{n_{2}, n_{4}, n_{6}\right\}\right)=H\left(n_{6}\right)$.

For $H\left(\left\{n_{2}, n_{4}, n_{6}\right\}\right)=H\left(n_{2}\right)$, the frequencies in $n_{4}$ and $n_{6}$ but not duplicated in $n_{1}, n_{2}$ and $n_{3}$ must be assigned to calls from $m_{2}, m_{3}$ or $m_{4}$. Thus we have $C\left(\left\{n_{4}, n_{6}\right\}\right)-r \leq s_{2}$, which implies that $2 i+2 j-c_{2}-c_{4}-r \leq 3 i+2 k$ (from Eq. (1) and (2)), i.e.,

$$
i+2 k+c_{2}+c_{4}+r \geq 2 j .
$$


As a result, $2 i+10 k+7 \sum_{i=1}^{4} c_{i}+7 r \geq 2\left(i+2 k+c_{2}+c_{4}+r\right)+3\left(2 k+c_{1}+c_{2}+c_{3}+r\right)+$ $4 c_{1}+2\left(c_{2}+c_{3}\right)+2\left(c_{3}+c_{4}\right)+3 c_{4}+2 r \geq 4 j$ because the first term is at least $4 j$ (from Eq. (4)) and all the other terms are non-negative (from Eq. (1) and (3)).

For $H\left(\left\{n_{2}, n_{4}, n_{6}\right\}\right)=H\left(n_{4}\right)$, the frequencies in $n_{2}$ and $n_{6}$ but not duplicated in $n_{3}, n_{4}$ and $n_{5}$ must be assigned to calls from $m_{6}, m_{7}$ or $m_{8}$. Thus we have $C\left(\left\{n_{2}, n_{6}\right\}\right)-r \leq s_{4}$, which implies that $2 i+2 j-c_{4}-r \leq 3 i+2 k+2 c_{2}+\min \left(c_{1}, c_{3}\right)$ (from Eq. (1) and (2)), i.e.,

$$
i+2 k+2 c_{2}+c_{4}+\min \left(c_{1}, c_{3}\right)+r \geq 2 j .
$$

As a result, $2 i+10 k+7 \sum_{i=1}^{4} c_{i}+7 r \geq 2\left(i+2 k+2 c_{2}+c_{4}+\min \left(c_{1}, c_{3}\right)+r\right)+3\left(2 k+c_{1}+\right.$ $\left.c_{2}+c_{3}+r\right)+4 c_{1}+2\left(c_{3}-\min \left(c_{1}, c_{3}\right)\right)+2\left(c_{3}+c_{4}\right)+3 c_{4}+2 r \geq 4 j$ because the first term is at least $4 j$ (from Eq. (5)) and all the other terms are non-negative (from Eq. (1) and $(3))$.

For $H\left(\left\{n_{2}, n_{4}, n_{6}\right\}\right)=H\left(n_{6}\right)$, the frequencies in $n_{2}$ and $n_{4}$ but not duplicated in $n_{1}, n_{5}$ and $n_{6}$ must be assigned to calls from $m_{10}, m_{11}$ or $m_{0}$. Thus we have $C\left(\left\{n_{2}, n_{4}\right\}\right)-r \leq s_{6}$, which implies that $2 i+2 j-c_{2}-r \leq 3 i+2 k+2 c_{4}+\min \left(0, c_{3}\right)$ (from Eq. (1) and (2)), i.e.,

$$
i+2 k+c_{2}+2 c_{4}+\min \left(0, c_{3}\right)+r \geq 2 j .
$$

As a result, $2 i+10 k+7 \sum_{i=1}^{4} c_{i}+7 r \geq 2\left(i+2 k+c_{2}+2 c_{4}+\min \left(0, c_{3}\right)+r\right)+3\left(2 k+c_{1}+\right.$ $\left.c_{2}+c_{3}+r\right)+4 c_{1}+2\left(c_{2}+c_{3}\right)+2\left(c_{3}-\min \left(0, c_{3}\right)\right)+3 c_{4} \geq 4 j$ because the first term is at least $4 j$ (from Eq. (6)) and all the other terms are non-negative (from Eq. (1) and (3)).

Thus, the lemma is proved for this case when $H\left(\left\{n_{1}, n_{2}, \ldots, n_{6}\right\}\right)=H\left(n_{1}\right)$.

Case 2: $\boldsymbol{H}\left(\left\{n_{1}, n_{2}, \ldots, n_{6}\right\}\right)=\boldsymbol{H}\left(n_{2}\right)$. Similar to the previous case, we can deduce that $C\left(\left\{n_{4}, n_{5}, n_{6}\right\}\right)-r \leq s_{2}$, i.e.,

$$
2 k+c_{2}+c_{3}+c_{4}+r \geq 2 j .
$$

Hence, $2 i+10 k+7 \sum_{i=1}^{4} c_{i}+7 r \geq 2 i+5\left(2 k+c_{2}+c_{3}+c_{4}+r\right)+5 c_{1}+2\left(c_{1}+c_{2}\right)+2\left(c_{3}+c_{4}\right)+2 r \geq 4 j$ because the second term is at least $10 j$ and all the other terms are non-negative.

For each of the remaining cases: we can also prove that $2 i+10 k+7 \sum_{i=1}^{4} c_{i}+7 r \geq 4 j$. The details are given in the appendix.

\section{Greedy in the With-Deletion Model}

In this section, we analyze Greedy in the with-deletion model, i.e., each call may be terminated at some time. We prove that the upper bound and lower bound of Greedy are both 3. Thus, the best possible competitive ratio is 3 for Greedy in the with-deletion model.

Theorem 3. Greedy for FAC is 3-competitive in the with-deletion model.

Proof. Consider Fig. 3(a), assume the highest frequency $f$ appears in cell $n_{0}$. Let $x$ be the number of frequencies in cell $n_{0}, y$ be the number of distinct frequencies in cells $n_{1}$ to $n_{6}$. Since Greedy is to choose the smallest frequency without interference with the neighboring frequencies, those frequencies less than $f$ must all appear in $n_{0}$ or $n_{i},(1 \leq i \leq 6)$. Therefore, we can say that 


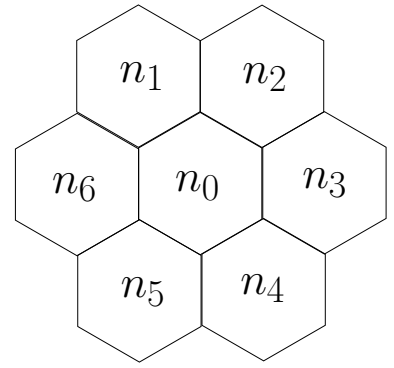

(a) Upper bound

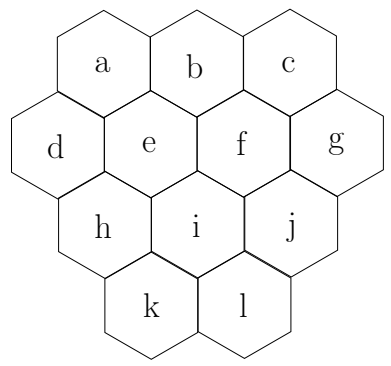

(b) Lower bound

Figure 3: Upper bound and Lower bound of Greedy in cellular network

$f=x+y$. In this configuration, optimal allocation uses at least $x+y / 3$ frequencies. Hence, the competitive ratio is at most

$$
\frac{x+y}{x+y / 3}<3 .
$$

The following is to prove by induction that 3-competitive is best possible for Greedy.

Consider Fig. 3(b), define tri-group to be the set of three cells which are "one edge" away from each other, e.g., cell sets $\{a, f, h\}$ and $\{b, g, i\}$.

We prove the following hypothesis by induction. When the highest frequency $3 n+1$ is assigned to a call in cell $x$, all frequencies from 1 to $3 n$ would have been used in a tri-group, e.g., $\{u, v, w\}$, around $x$, s.t. $C(u)=C(v)=C(w)=n$. As for the optimal off-line algorithm, $n+1$ frequencies could be sufficient to take up all these calls.

Base Step $n=1$. When one call appears at cells $c, d$ and $i$, Greedy uses the frequency 1 to these calls. Then the frequency 2 will be assigned to a call at cells $a$ and cell $g$, and the frequency 3 to a call at cell $b$. Delete the call in cell $c$. At last, the frequency 4 has to be assigned to a call at cell $f$, since the frequencies 1,2 and 3 are all around $f$.

In this step, Greedy uses 4 frequencies while the optimal off-line assignment uses only two frequencies, and each cell of tri-group $\{b, g, i\}$ contains one frequency.

Induction Step Suppose the hypothesis is true for $n=k$. Now we prove that it is also correct for $n=k+1$.

By the induction hypothesis, Greedy can force the frequency $3 k+1$ to a call $C_{1}$ in cell $d$ and another call $C_{2}$ in cell $g$. Terminate all other calls except $C_{1}$ and $C_{2}$. From the hypothesis, Greedy can assign the frequencies 1 to $3 k$ to appear in tri-group $\{a, f, h\}$, and each cell contains $k$ frequencies. Then a call $C_{3}$ arrives at $e$, Greedy must use the frequency $3 k+2$. Then terminate all other calls except $C_{3}$ in $e$ and $C_{2}$ in $g$. After that, Greedy forces the frequencies 1 to $3 k$ to appear in tri-group $\{b, g, i\}$, s.t. $C(b)=C(i)=k$ and $C(g)=k+1$ (including the call $C_{2}$ ). Now a call $C_{4}$ arrives at $f$, Greedy must use the frequency $3 k+3$ to this call. Then terminate all other calls except $C_{4}$ in $f$. By similar description, Greedy can assign the frequency $3 k+1$ to a call $C_{5}$ in $h$ and the frequency $3 k+2$ to a call $C_{6}$ in $l$. Then force the frequencies 1 to $3 k$ to appear in tri-group $\{f, h, l\}$, s.t. $C(f)=C(h)=C(l)=3 k+1$. Finally, one call $C_{7}$ arrives at $i$, Greedy must use the frequency $3 k+4$. 
With the induction hypothesis on the above sequence, the optimal off-line algorithm can assign frequencies $k+1, k+2, k+2, k+1, k+1, k+1$ and $k+2$ to the calls $C_{1}$ to $C_{7}$ respectively.

Thus, to satisfy the call sequence described above, Greedy must use $3 k+4$ frequencies, while the optimal off-line assignment uses only $k+2$ frequencies. Therefore, the competitive ratio of Greedy in cellular network is at least

$$
\frac{3 n-2}{n} \longrightarrow 3
$$

\section{References}

[1] K. I. Aardal, S. P. M. van Hoesel, A. M. C. A. Koster, C. Mannino, and A. Sassano. Models and solution techniques for frequency assignment problems. Quarterly Journal of the Belgian, French and Italian Operations Research Societies (4OR), 1(4):261-317, 2003.

[2] A. Borodin and R. El-Yaniv. Online Computation and Competitive Analysis. Cambridge University Press, 1998.

[3] I. Caragiannis, C. Kaklamanis, and E. Papaioannou. Efficient on-line frequency allocation and call control in cellular networks. Theory Comput. Syst., 35(5):521-543, 2002. A preliminary version of the paper appeared in SPAA 2000.

[4] W. Hale. Frequency assignment: Theory and applications. Proceedings of the IEEE, 68(12):1497-1514, 1980.

[5] J. Janssen, D. Krizanc, L. Narayanan, and S. M. Shende. Distributed online frequency assignment in cellular networks. J. Algorithms, 36(2):119-151, 2000.

[6] B. Jaumard, O. Marcotte, and C. Meyer. Mathematical models and exact methods for channel assignment in cellular networks. In B. Sansò and P. Soriano, editors, Telecommunications Network Planning, pages 239-255. Kluwer, 1999.

[7] I. Katzela and M. Naghshineh. Channel assignment schemes for cellular mobile telecommunication systems: A comprehensive survey. IEEE Personal Communications, 3(3):10-31, 1996.

[8] V. H. MacDonald. The cellular concept. The Bell System Techn. J. 58: 15-41, 1979

[9] C. McDiarmid and B. A. Reed. Channel assignment and weighted coloring. Networks, $36(2): 114-117,2000$

[10] L. Narayanan. Channel assignment and graph multicoloring. In I. Stojmenović, editor, Handbook of Wireless Networks and Mobile Computing, pages 71-94. John Wiley \& Sons, 2002. 


\section{Appendix}

\section{Remaining proof of Lemma 2}

We continue for the remaining cases, i.e., $H\left(\left\{n_{1}, n_{2}, \ldots, n_{6}\right\}\right)=H\left(n_{i}\right)$ for $3 \leq i \leq 6$.

Case 3: $\boldsymbol{H}\left(\left\{n_{1}, n_{2}, \ldots, n_{6}\right\}\right)=\boldsymbol{H}\left(n_{3}\right)$. Similar to Case 1, we have

$$
\begin{aligned}
& C\left(\left\{n_{1}, n_{5}, n_{6}\right\}\right)-r \leq s_{3}=3 i+j+2 k+2 c_{1} \\
\Rightarrow \quad & 3 i+j-c_{3}-c_{4}-r \leq 3 i+j+2 k+2 c_{1} \\
\Rightarrow \quad & 2 k+2 c_{1}+c_{3}+c_{4}+r \geq 0 .
\end{aligned}
$$

We further consider three sub-cases: $H\left(\left\{n_{2}, n_{4}, n_{6}\right\}\right)=H\left(n_{2}\right), H\left(\left\{n_{2}, n_{4}, n_{6}\right\}\right)=H\left(n_{4}\right)$, and $H\left(\left\{n_{2}, n_{4}, n_{6}\right\}\right)=H\left(n_{6}\right)$.

For $H\left(\left\{n_{2}, n_{4}, n_{6}\right\}\right)=H\left(n_{2}\right)$, as in case 1 , we have $i+2 k+c_{2}+c_{4}+r \geq 2 j$. As a result, $2 i+10 k+7 \sum_{i=1}^{4} c_{i}+7 r \geq 2\left(i+2 k+c_{2}+c_{4}+r\right)+3\left(2 k+2 c_{1}+c_{3}+c_{4}+r\right)+\left(c_{1}+c_{2}\right)+$ $4\left(c_{2}+c_{3}\right)+2 c_{4}+2 r \geq 4 j$ because the first term is at least $4 j$ and all the other terms are non-negative.

For $H\left(\left\{n_{2}, n_{4}, n_{6}\right\}\right)=H\left(n_{4}\right)$, as in case 1 , we have $i+2 k+2 c_{2}+c_{4}+\min \left(c_{1}, c_{3}\right)+r \geq 2 j$. As a result, $2 i+10 k+7 \sum_{i=1}^{4} c_{i}+7 r \geq 2\left(i+2 k+2 c_{2}+c_{4}+\min \left(c_{1}, c_{3}\right)+r\right)+3\left(2 k+2 c_{1}+\right.$ $\left.c_{3}+c_{4}+r\right)+\left(c_{1}+c_{3}-2 \min \left(c_{1}, c_{3}\right)\right)+3\left(c_{2}+c_{3}\right)+2 c_{4}+2 r \geq 4 j$ because the first term is at least $4 j$ and all the other terms are non-negative.

For $H\left(\left\{n_{2}, n_{4}, n_{6}\right\}\right)=H\left(n_{6}\right)$, as in case 1 , we have $i+2 k+c_{2}+2 c_{4}+\min \left(0, c_{3}\right)+r \geq 2 j$. As a result, $2 i+10 k+7 \sum_{i=1}^{4} c_{i}+7 r \geq 2\left(i+2 k+c_{2}+2 c_{4}+\min \left(0, c_{3}\right)+r\right)+3\left(2 k+2 c_{1}+\right.$ $\left.c_{3}+c_{4}+r\right)+\left(c_{1}+c_{2}\right)+4\left(c_{2}+c_{3}\right)-2 \min \left(0, c_{3}\right)+2 r \geq 4 j$ because the first term is at least $4 j$ and all the other terms are non-negative.

Case 4: $\boldsymbol{H}\left(\left\{n_{1}, n_{2}, \ldots, n_{6}\right\}\right)=\boldsymbol{H}\left(n_{4}\right)$. Similar to the previous cases, we can deduce that $C\left(\left\{n_{1}, n_{2}, n_{6}\right\}\right)-r \leq s_{4}=3 i+2 k+2 c_{2}+\min \left\{c_{1}, c_{3}\right\}$, i.e.,

$$
2 k+2 c_{2}+c_{4}+\min \left(c_{1}, c_{3}\right)+r \geq 2 j .
$$

We further consider three sub-cases: $H\left(\left\{n_{1}, n_{3}, n_{5}\right\}\right)=H\left(n_{1}\right), H\left(\left\{n_{1}, n_{3}, n_{5}\right\}\right)=H\left(n_{3}\right)$, and $H\left(\left\{n_{1}, n_{3}, n_{5}\right\}\right)=H\left(n_{5}\right)$.

For $H\left(\left\{n_{1}, n_{3}, n_{5}\right\}\right)=H\left(n_{1}\right)$, the frequencies in $n_{3}$ and $n_{5}$ but not duplicated in $n_{1}, n_{2}$ and $n_{6}$ must be assigned to calls from $m_{0}, m_{1}$ or $m_{2}$. Thus we have $C\left(\left\{n_{3}, n_{5}\right\}\right)-r \leq s_{1}$, which implies that $2 i-c_{1}-c_{3}-r \leq 3 i+j+2 k$, i.e.,

$$
i+2 k+c_{1}+c_{3}+r \geq-j .
$$

As a result, $2 i+10 k+7 \sum_{i=1}^{4} c_{i}+7 r \geq 3\left(2 k+2 c_{2}+c_{4}+\min \left(c_{1}, c_{3}\right)+r\right)+2\left(i+2 k+c_{1}+\right.$ $\left.c_{3}+r\right)+2 c_{1}+3\left(c_{1}-\min \left(c_{1}, c_{3}\right)\right)+\left(c_{2}+c_{3}\right)+4\left(c_{3}+c_{4}\right)+2 r \geq 4 j$ because the first term is at least $6 j$, the second term is at least $-2 j$ and all the other terms are non-negative. 
For $H\left(\left\{n_{1}, n_{3}, n_{5}\right\}\right)=H\left(n_{3}\right)$, the frequencies in $n_{1}$ and $n_{5}$ but not duplicated in $n_{2}, n_{3}$ and $n_{4}$ must be assigned to calls from $m_{4}, m_{5}$ or $m_{6}$. Thus we have $C\left(\left\{n_{1}, n_{5}\right\}\right)-r \leq s_{3}$, which implies that $2 i-c_{3}-r \leq 3 i+j+2 k+2 c_{1}$, i.e.,

$$
i+2 k+2 c_{1}+c_{3}+r \geq-j .
$$

As a result, $2 i+10 k+7 \sum_{i=1}^{4} c_{i}+7 r \geq 3\left(2 k+2 c_{2}+c_{4}+\min \left(c_{1}, c_{3}\right)+r\right)+2\left(i+2 k+2 c_{1}+\right.$ $\left.c_{3}+r\right)+3\left(c_{1}-\min \left(c_{1}, c_{3}\right)\right)+\left(c_{2}+c_{3}\right)+4\left(c_{3}+c_{4}\right)+2 r \geq 4 j$ because the first term is at least $6 j$, the second term is at least $-2 j$ and all the other terms are non-negative.

For $H\left(\left\{n_{1}, n_{3}, n_{5}\right\}\right)=H\left(n_{5}\right)$, the frequencies in $n_{1}$ and $n_{3}$ but not duplicated in $n_{4}, n_{5}$ and $n_{6}$ must be assigned to calls from $m_{8}, m_{9}$ or $m_{10}$. Thus we have $C\left(\left\{n_{1}, n_{3}\right\}\right)-r \leq s_{5}$, which implies that $2 i-c_{1}-r \leq 3 i+j+2 k+2 c_{3}+\min \left(c_{2}, c_{4}\right)$, i.e.,

$$
i+2 k+c_{1}+2 c_{3}+\min \left(c_{2}, c_{4}\right)+r \geq-j .
$$

As a result, $2 i+10 k+7 \sum_{i=1}^{4} c_{i}+7 r \geq 3\left(2 k+2 c_{2}+c_{4}+\min \left(c_{1}, c_{3}\right)+r\right)+2\left(i+2 k+c_{1}+2 c_{3}+\right.$ $\left.\min \left(c_{2}, c_{4}\right)+r\right)+4 c_{1}+\left(c_{1}+c_{2}\right)+2 c_{4}+3\left(c_{3}-\min \left(c_{1}, c_{3}\right)\right)+2\left(c_{4}-\min \left(c_{2}, c_{4}\right)\right)+2 r \geq 4 j$ because the first term is at least $6 j$, the second term is at least $-2 j$ and all the other terms are non-negative.

Case 5: $\boldsymbol{H}\left(\left\{n_{1}, n_{2}, \ldots, n_{6}\right\}\right)=\boldsymbol{H}\left(n_{5}\right)$. Similar to the previous cases, we can deduce that $C\left(\left\{n_{1}, n_{2}, n_{3}\right\}\right)-r \leq s_{5}=3 i+j+2 k+2 c_{3}+\min \left(c_{2}, c_{4}\right)$, i.e.,

$$
2 k+c_{1}+2 c_{3}+\min \left(c_{2}, c_{4}\right)+r \geq 0 .
$$

We further consider three sub-cases: $H\left(\left\{n_{2}, n_{4}, n_{6}\right\}\right)=H\left(n_{2}\right), H\left(\left\{n_{2}, n_{4}, n_{6}\right\}\right)=H\left(n_{4}\right)$, and $H\left(\left\{n_{2}, n_{4}, n_{6}\right\}\right)=H\left(n_{6}\right)$.

For $H\left(\left\{n_{2}, n_{4}, n_{6}\right\}\right)=H\left(n_{2}\right)$, as in the previous cases, we have $i+2 k+c_{2}+c_{4}+r \geq 2 j$. As a result, $2 i+10 k+7 \sum_{i=1}^{4} c_{i}+7 r \geq 2\left(i+2 k+c_{2}+c_{4}+r\right)+3\left(2 k+c_{1}+2 c_{3}+\min \left(c_{2}, c_{4}\right)+\right.$ $r)+4\left(c_{1}+c_{2}\right)+\left(c_{2}+c_{3}\right)+3\left(c_{4}-\min \left(c_{2}, c_{4}\right)\right)+2 c_{4}+2 r \geq 4 j$ because the first term is at least $4 j$ and all the other terms are non-negative.

For $H\left(\left\{n_{2}, n_{4}, n_{6}\right\}\right)=H\left(n_{4}\right)$, as in the previous cases, we have $i+2 k+2 c_{2}+c_{4}+\min \left(c_{1}, c_{3}\right)+r \geq$ $2 j$. As a result, $2 i+10 k+7 \sum_{i=1}^{4} c_{i}+7 r \geq 2\left(i+2 k+2 c_{2}+c_{4}+\min \left(c_{1}, c_{3}\right)+r\right)+3\left(2 k+c_{1}+\right.$ $\left.2 c_{3}+\min \left(c_{2}, c_{4}\right)+r\right)+2\left(c_{1}-\min \left(c_{1}, c_{3}\right)\right)+2 c_{1}+3\left(c_{2}-\min \left(c_{2}, c_{4}\right)\right)+\left(c_{3}+c_{4}\right)+4 c_{4}+2 r \geq 4 j$ because the first term is at least $4 j$ and all the other terms are non-negative.

For $H\left(\left\{n_{2}, n_{4}, n_{6}\right\}\right)=H\left(n_{6}\right)$, as in the previous cases, we have $i+2 k+c_{2}+2 c_{4}+\min \left(0, c_{3}\right)+r \geq$ $2 j$. As a result, $2 i+10 k+7 \sum_{i=1}^{4} c_{i}+7 r \geq 2\left(i+2 k+c_{2}+2 c_{4}+\min \left(0, c_{3}\right)+r\right)+3\left(2 k+c_{1}+2 c_{3}+\right.$ $\left.\min \left(c_{2}, c_{4}\right)+r\right)+2\left(c_{1}-\min \left(0, c_{3}\right)\right)+2\left(c_{1}+c_{2}\right)+3\left(c_{2}-\min \left(c_{2}, c_{4}\right)\right)+\left(c_{3}+c_{4}\right)+2 c_{4}+2 r \geq 4 j$ because the first term is at least $4 j$ and all the other terms are non-negative.

Case 6: $\boldsymbol{H}\left(\left\{n_{1}, n_{2}, \ldots, n_{6}\right\}\right)=\boldsymbol{H}\left(n_{6}\right)$. Similar to the previous cases, we can deduce that $C\left(\left\{n_{2}, n_{3}, n_{4}\right\}\right)-r \leq s_{6}=3 i+2 k+2 c_{4}+\min \left(0, c_{3}\right)$, i.e.,

$$
2 k+c_{1}+c_{2}+2 c_{4}+\min \left(0, c_{3}\right)+r \geq 2 j .
$$

We further consider three sub-cases: $H\left(\left\{n_{1}, n_{3}, n_{5}\right\}\right)=H\left(n_{1}\right), H\left(\left\{n_{1}, n_{3}, n_{5}\right\}\right)=H\left(n_{3}\right)$, and $H\left(\left\{n_{1}, n_{3}, n_{5}\right\}\right)=H\left(n_{5}\right)$. 
For $H\left(\left\{n_{1}, n_{3}, n_{5}\right\}\right)=H\left(n_{1}\right)$, as in the previous cases, we have $i+2 k+c_{1}+c_{3}+r \geq-j$.

As a result, $2 i+10 k+7 \sum_{i=1}^{4} c_{i}+7 r \geq 3\left(2 k+c_{1}+c_{2}+2 c_{4}+\min \left(0, c_{3}\right)+r\right)+2(i+2 k+$ $\left.c_{1}+c_{3}+r\right)+2\left(c_{1}+c_{2}\right)+2\left(c_{2}+c_{3}\right)+3\left(c_{3}-\min \left(0, c_{3}\right)\right)+c_{4}+2 r \geq 4 j$ because the first term is at least $6 j$, the second term is at least $-2 j$ and all the other terms are non-negative.

For $H\left(\left\{n_{1}, n_{3}, n_{5}\right\}\right)=H\left(n_{3}\right)$, as in the previous cases, we have $i+2 k+2 c_{1}+c_{3}+r \geq-j$.

As a result, $2 i+10 k+7 \sum_{i=1}^{4} c_{i}+7 r \geq 3\left(2 k+c_{1}+c_{2}+2 c_{4}+\min \left(0, c_{3}\right)+r\right)+2(i+2 k+$ $\left.2 c_{1}+c_{3}+r\right)+4\left(c_{2}+c_{3}\right)+\left(c_{3}+c_{4}\right)-3 \min \left(0, c_{3}\right)+2 r \geq 4 j$ because the first term is at least $6 j$, the second term is at least $-2 j$ and all the other terms are non-negative.

For $H\left(\left\{n_{1}, n_{3}, n_{5}\right\}\right)=H\left(n_{5}\right)$, as in the previous cases, we have $i+2 k+c_{1}+2 c_{3}+\min \left(c_{2}, c_{4}\right)+r \geq$ $-j$.

As a result, $2 i+10 k+7 \sum_{i=1}^{4} c_{i}+7 r \geq 3\left(2 k+c_{1}+c_{2}+2 c_{4}+\min \left(0, c_{3}\right)+r\right)+2\left(i+2 k+c_{1}+\right.$ $\left.2 c_{3}+\min \left(c_{2}, c_{4}\right)+r\right)+2\left(c_{1}+c_{2}\right)+2\left(c_{2}-\min \left(c_{2}, c_{4}\right)\right)+3\left(c_{3}-\min \left(0, c_{3}\right)\right)+c_{4}+2 r \geq 4 j$ because the first term is at least $6 j$, the second term is at least $-2 j$ and all the other terms are non-negative. 